\title{
Detecting non-random mating or selection in natural populations using multi-locus gene families
}

\author{
Gabe O'Reilly ${ }^{1}$, Oliver Manlik ${ }^{2}$, Sandra Vardeh $^{3}$, Jennifer Sinclair ${ }^{4}$, Zachary Lawler ${ }^{5}$, and \\ William Sherwin ${ }^{1}$ \\ ${ }^{1}$ University of New South Wales Faculty of Science \\ ${ }^{2}$ UAE University \\ ${ }^{3}$ Bundesamt für Naturschutz \\ ${ }^{4}$ Cape Bernier Vineyard \\ ${ }^{5}$ The University of Newcastle
}

May 6, 2021

\begin{abstract}
New sequencing technologies have opened the door to many new research opportunities, but these advances in data collection are not always compatible with some important methods for data analysis. Fis has been a staple calculation in the field of population genetics. Fis can be used to measure either a departure from random mating, or measure underlying selective pressures for or against heterozygote genotypes. However, when using Next Generation Sequencing (NGS) technology on multi-locus gene families it is often impossible to discern which allelic variants are present at each locus. Some important multi-locus gene families are: the major histocompatibility complex (MHC) in animals; homeobox genes in fungi; or the self-incompatibility genes in plants. This in turn makes it impossible to calculate either locus-specific expected heterozygosity, or observed heterozygosity, both of which are required to calculate Fis. Without the ability to calculate Fis from NGS of multi-locus gene families, we need a new multi-locus measure that will allow us to detect the underlining mating, and selective patterns present in such multi-locus genes. This paper provides such a novel multi-locus measure, called 1His. We demonstrate the accuracy of the 1 His equation using simulated data, and two datasets taken from natural populations of dolphins and penguins. The introduction of this new measure is particularly important because of the great interest in mating patterns and selection of multi-locus gene families, such as MHC.
\end{abstract}

\section{Hosted file}

Inbreeding paper - MolEcol.pdf available at https://authorea.com/users/412331/articles/ 521050-detecting-non-random-mating-or-selection-in-natural-populations-using-multilocus-gene-families 